\title{
Direct bare metal needle puncture and balloon angioplasty in calcified plaques of the common femoral artery guided by angiography ("BAMBOO SPEAR")
}

Naoki Hayakawa ${ }^{1 *}$ (D), Satoshi Kodera ${ }^{2}$, Masataka Arakawa ${ }^{1}$, Satoshi Hirano ${ }^{1}$, Sandeep Shakya ${ }^{1}$ and Junji Kanda ${ }^{1}$

\begin{abstract}
Background: Surgical endarterectomy for common femoral artery (CFA) disease is still considered the gold standard for treatment. Development of various techniques and devices has improved the clinical results of endovascular therapy (EVT) for CFA. However, severe conditions remain, especially for occlusive lesions owing to calcified plaque. We developed a useful technique for passing a lesion by directly penetrating the calcified plaque of the CFA using a bare metal needle and then passing through a balloon or dilating it. We named this technique "direct bare metal needle puncture and balloon angioplasty in calcified plaques of the common femoral artery guided by angiography" or "BAMBOO SPEAR."
\end{abstract}

Main text: This report describes our technique for crossing a lesion by directly penetrating the calcified plaque of the CFA using a needle. We report a case of a 73-year-old male with hemodialysis who presented with cyanosis and ischemic rest pain of both lower limbs. Control angiography showed total occlusion of the left CFA with a calcified plaque. We advanced a 21-G metal needle that was slightly curved into the blood vessel from where the lumen of the distal CFA was located. The needle was advanced into the center of the calcified plaque, while observing from multiple directions with a fluoroscopic guide. We succeeded in advancing the needle into the lumen of the distal external iliac artery. After guidewire crossing, intravascular ultrasound (IVUS) showed that guidewire was able to completely pass through the center of the calcified plaque. We could dilate the lesion by scoring balloon and drug-coated balloon. The final angiography showed sufficient results. We named this technique "direct BAre Metal needle puncture and BallOOn angioplaSty in calcified PlaquEs of the common femoral ARtery guided by angiography" (BAMBOO SPEAR).

Conclusions: The BAMBOO SPEAR technique may be considered a useful option in EVT for occlusive CFA with calcified plaques.

Keywords: Endovascular therapy, Needle, Common femoral artery, Calcified plaque

\footnotetext{
* Correspondence: haya.naoki1981@gmail.com

'Department of Cardiovascular Medicine, Asahi General Hospital, I-1326

Asahi, Chiba 289-2511, Japan

Full list of author information is available at the end of the article
}

\section{Springer Open}

(c) The Author(s). 2021 Open Access This article is licensed under a Creative Commons Attribution 4.0 International License, which permits use, sharing, adaptation, distribution and reproduction in any medium or format, as long as you give appropriate credit to the original author(s) and the source, provide a link to the Creative Commons licence, and indicate if changes were made. The images or other third party material in this article are included in the article's Creative Commons licence, unless indicated otherwise in a credit line to the material. If material is not included in the article's Creative Commons licence and your intended use is not permitted by statutory regulation or exceeds the permitted use, you will need to obtain permission directly from the copyright holder. To view a copy of this licence, visit http://creativecommons.org/licenses/by/4.0/. 


\section{Background}

Development of endovascular therapy (EVT) led to one of the first-line treatment strategies for peripheral artery disease (PAD) (Norgen et al. 2007). However, surgical endarterectomy is still considered as the gold standard treatment in common femoral artery (CFA) disease because of favorable long-term durability (Nishibe et al. 2015). However, postoperative morbidity, including wound infection and lymphatic leakage, can occur after this surgical approach (Nguyen et al. 2015). Recently, feasible clinical results of stenting for the CFA or EVT for the CFA using a drug coated balloon (DCB) have been reported (Kuo et al. 2019; Gouëffic et al. 2017). However, if severe calcified lesions or eccentric calcified plaques are present, dilatation is often difficult. Excessive dilation may lead to a risk of vascular perforation, which leads to the need for using stent-grafts. We sometimes intentionally advance the extra hard tip of a guidewire through the center of calcification, but it can be difficult for severe calcifications. The use of atherectomy devices can be useful, but there are also problems with this device, such as the fact that some areas do not cover medical insurance for this device, the high cost, and complexity of the procedure (Stavroulakis et al. 2018). We developed a useful and simple technique for crossing a lesion by directly penetrating the calcified plaque of the CFA using a bare metal needle, which then passes through the balloons or dilates it. After passing through the guidewire, externalization of the guidewire is performed, and then a bougie is used with a needle so that balloons can pass through relatively easily. Because the guidewire can pass through the center of a calcified plaque, the risk of vascular injury is small and the balloon can be well dilated, and an acceptable initial lumen gain is easy to obtain. We named this technique "direct BAre Metal needle puncture and BallOOn angioplaSty in calcified PlaquEs of the common femoral ARtery guided by angiography" (BAMBOO SPEAR). And we present here a case of using this BAMBOO SPEAR technique for total occlusion of the CFA with calcified plaques.

\section{Main text}

A 73-year-old man had hemodialysis owing to diabetes mellitus with coronary artery disease and old cerebral infarction. Cyanosis and ischemic rest pain of both lower limbs were observed. Preprocedural plain computed tomography showed severe calcification in the left femoral artery (Fig. 1a). A 6-Fr Destination guiding sheath was inserted into the right CFA via the contralateral approach. Control angiography showed total occlusion of the left CFA with a calcified plaque and total occlusion of the middle to distal SFA (Fig. 1b-d). Because calcification was remarkable, we decided to use a metal needle from the retrograde direction in primary. A 21-G metal needle (Merit Advance ${ }^{\bullet}$ angiography needle; Merit Medical) that was slightly curved was inserted into the blood vessel from where the lumen of the distal CFA was located. The needle was advanced into the center of the calcified plaque, while observing from multiple directions with a fluoroscopic guide (Fig. 2a, b). We succeeded in advancing the needle as it was and into the lumen of the distal external iliac artery (Fig. 2c). A 0.014-in. guidewire (Gladius MGES $^{\oplus}$ guidewire; Asahi Intec) was inserted from behind the needle and the guidewire was advanced into the guiding sheath to
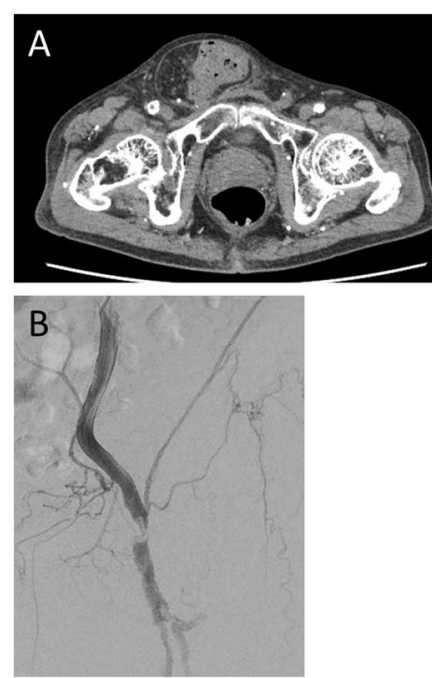

Fig. 1 a Plain computed tomography scan shows a calcified plaque in the left CFA. b-d Control angiography shows total occlusion of the left CFA with a calcified plaque and total occlusion of the middle to distal SFA

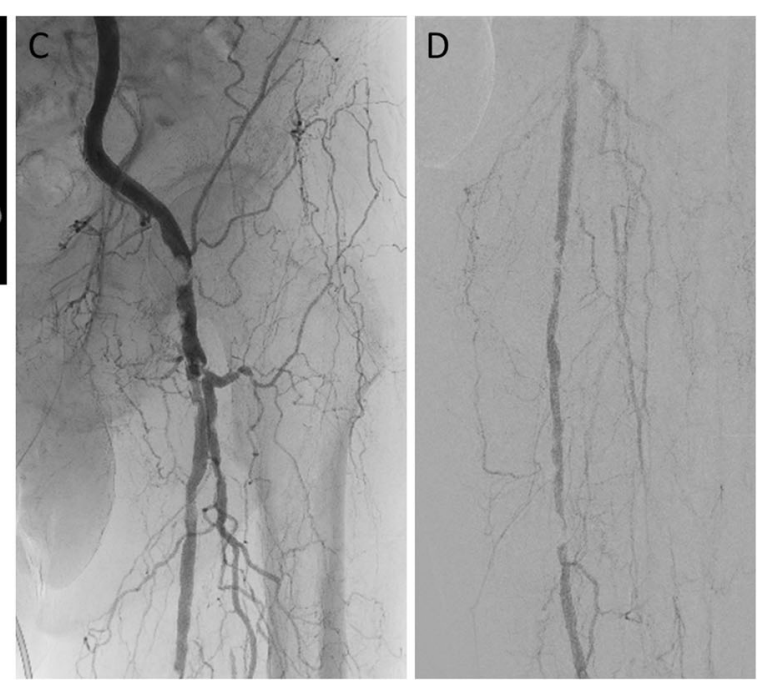




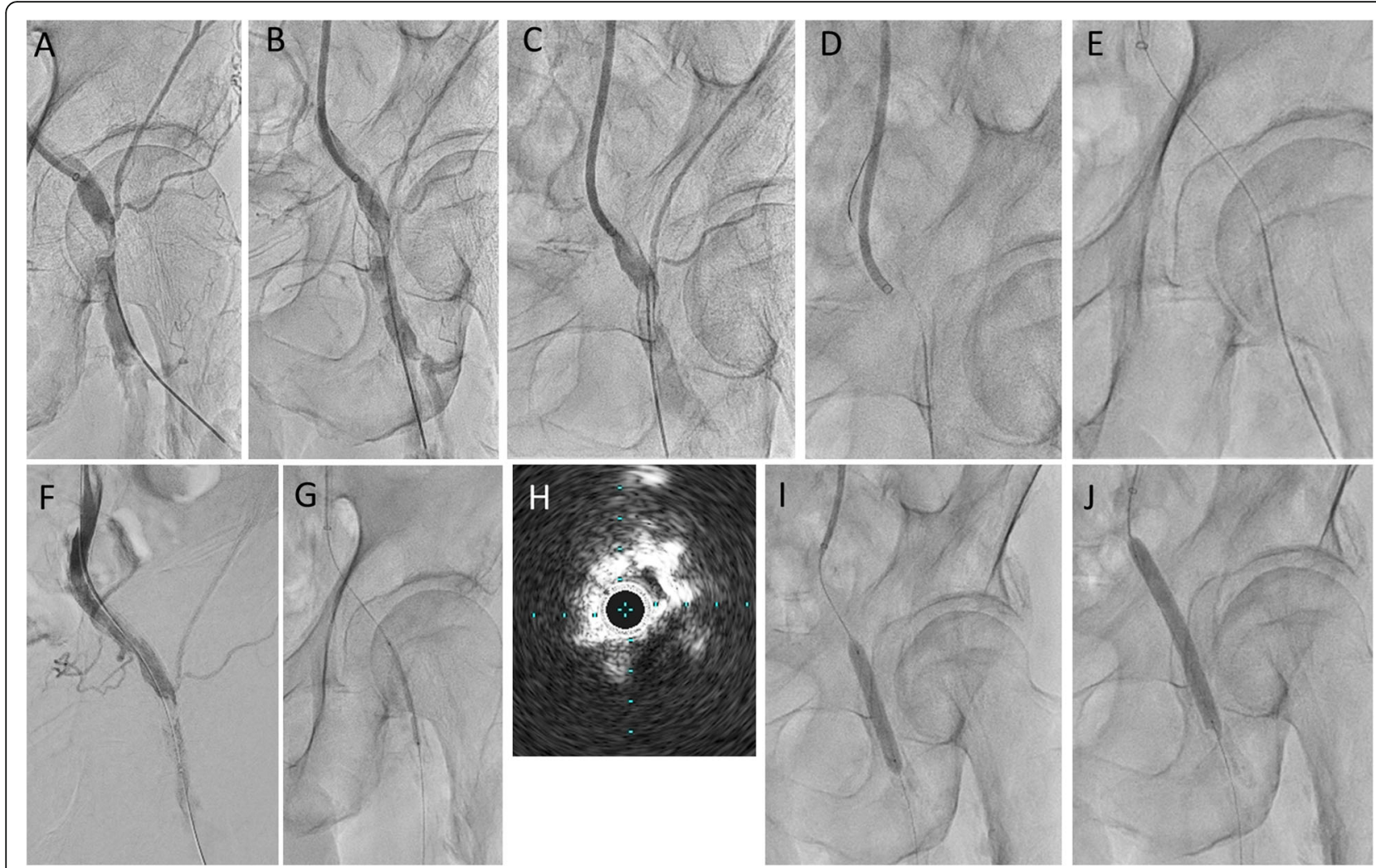

Fig. 2 a, b Direct puncture from the distal CFA. c We succeeded in advancing the needle as it was and into the lumen of the distal external iliac artery. $\mathbf{d}-\mathbf{f}$ A 0.014 -in. guidewire was inserted from behind the needle and the guidewire was advanced into the guiding sheath to make it externalized. $\mathbf{g}$ We dilated a $3.0 \times 40-\mathrm{mm}$ balloon. $\mathbf{h}$ IVUS shows the guidewire passed through the center of a calcified plaque. $\mathbf{i}, \mathbf{j}$ We dilated the scoring balloon and drug-coated balloon

enable its externalization (Fig. 2d-f). The calcified plaque was bougied several times with a metal needle that was coaxially placed on the externalization wire to perform modification of the lesion. After the antegrade re-wiring and pre dilatation, IVUS showed that the wire route was able to completely pass through the center of the calcified plaque (Fig. 2g, h). After dilation with a scoring balloon $6.0 \times 40$-mm (Lacrosse $\mathrm{NSE}^{\oplus}$ scoring balloon; Nippro) with intravascular hemostasis of the retrograde puncture site (Fig. 2i). Finally, we dilated a $6.0 \times$ 60-mm drug-coated balloon (Lutonix $\mathrm{RX}^{\circ}$ drug-coated balloon; BARD, AZ, USA) at the CFA lesion to prevent restenosis (Fig. 2j). The final angiography showed good results and the pressure gradient had disappeared completely (Fig. 3a, b). When the residual lesion of the SFA was treated 5 months after intervention of the CFA, the CFA was well opened, and IVUS also showed a sufficient lumen area (Fig. 3c-f).

\section{BAMBOO SPEAR technique}

A slightly curved 21-G or 20-G metal needle was inserted from distal true lumen and then advanced into the center of the calcified plaque, while observing from multiple directions with a fluoroscopic guide (Fig. 4a-d). The metal needle on the externalized guidewire was then coaxially placed in and out several times to puncture the lesion (Fig. 4e, f). We then advanced another guidewire in the direction of the SFA. We usually placed a filter wire in the popliteal artery (Fig. 4g). The lesion was dilated with an antegrade balloon. At this time, the metal needle was removed, but by covering and expanding the puncture site, hemostasis could be achieved from inside the blood vessel at the same time (Fig. 4h). Using the results of angiography after balloon dilatation, we decided to finish with conventional balloon angioplasty alone, a DCB, or deploying a stent if recoil or dissection was severe (Fig. 4i, j).

\section{Discussion}

We demonstrated the feasibility of EVT for CFA with severe calcified plaques using direct puncture of a metal needle. In this case, we were able to pass through the center of the calcified plaque and obtain sufficient initial lumen gain after balloon angioplasty by a simple method. Obtaining the initial lumen area is difficult if severe calcified lesions are present. Especially in the case 

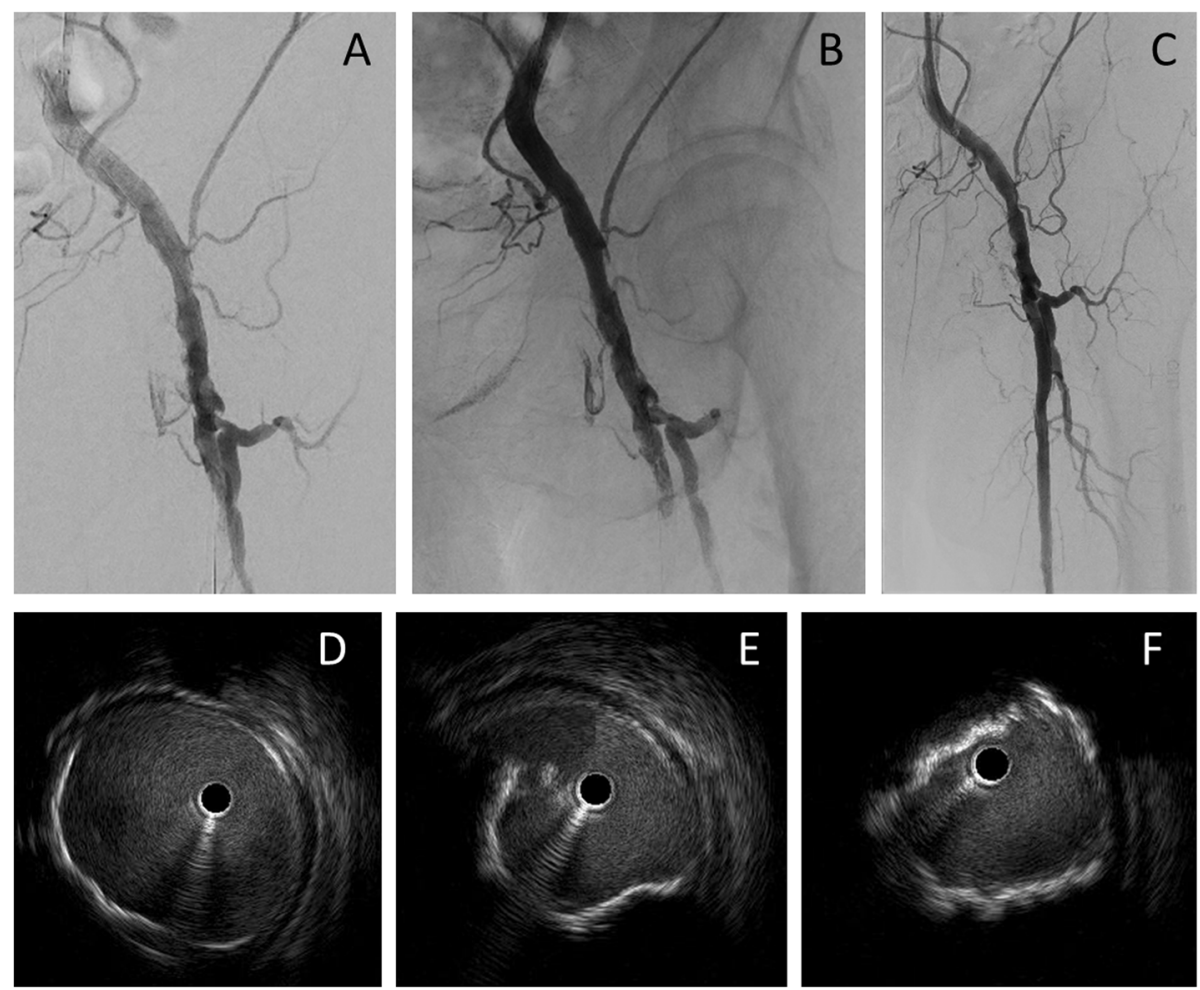

Fig. $\mathbf{3}$ a, b Final angiography shows sufficient expansion of the lesions. c Angiography when the SFA was treated 5 months after CFA treatment. The CFA was well opened. $\mathbf{d}$-f IVUS shows that the CFA is sufficiently patent. Panel $\mathbf{d}$ shows the proximal CFA, panel e shows the mid-CFA, and panel c shows the distal CFA

of eccentric calcified nodules, not only does an open lumen not spread sufficiently, but excessive balloon dilation also involves the risk of vascular perforation. There has been a report on the feasibility of atherectomy devices (Stavroulakis et al. 2018). However, there is also the problem that these devices cannot be used in some areas for the reason that medical insurance does not cover them.

Passing the guidewire through heavy calcification is often difficult. Our method appears to be a simple and easy method of piercing a lesion with a metal needle and modifying the lesion. In the EVT field, methods using a metal needle, such as the PIERCE technique, and inner PIERCE technique for patients in whom there is difficulty in passing a balloon, have been reported (Ichihashi et al. 2014; Nakama et al. 2020). Metal needles are much harder and sharper than guidewires, and therefore, they can penetrate even with severe calcification. Our method is primarily to use a metal needle instead of guidewires to allow severe calcification to pass directly. Our method can also help subsequent balloon passage by modifying the lesion from intravessels, as well as passage of calcification.

We usually use a scoring balloon or cutting balloon after guidewire passage combined with high-pressure balloons.
Surgical endarterectomy appears to be a gold standard treatment for calcified CFA lesions. However, we consider that our method can be indicated for cases in which EVT should be chosen because of problems, such as the general condition and comorbidities. Additionally, our method can be indicated in cases in which performing EVT appears to be preferable for aortoiliac lesions and femoropopliteal lesions at the same time as CFA disease.

As a possible complication, the possibility of distal embolism cannot be ruled out because calcification expands while being crushed. Therefore, we usually try to place a filter wire (Parachute ${ }^{\circ}$ filter wire; Keisei Medical, Tokyo, Japan) in the popliteal artery after passing the anterograde guidewire. Fortunately, we have not experienced any clinically problematic distal embolism. We always insert a guidewire into the antegrade guiding sheath to make the wire externalized. This procedure reduces the risk that the guidewire will be under tension and the needle will get caught. Enlarging the fluoroscopic image and attempting to proceed slowly at the beginning as much as possible are also important to avoid complications. Advancing carefully with the metal needle and guide wire as coaxially as possible is also recommended.

In our case, the initial success of the procedure and good short-term prognosis were confirmed, but the 


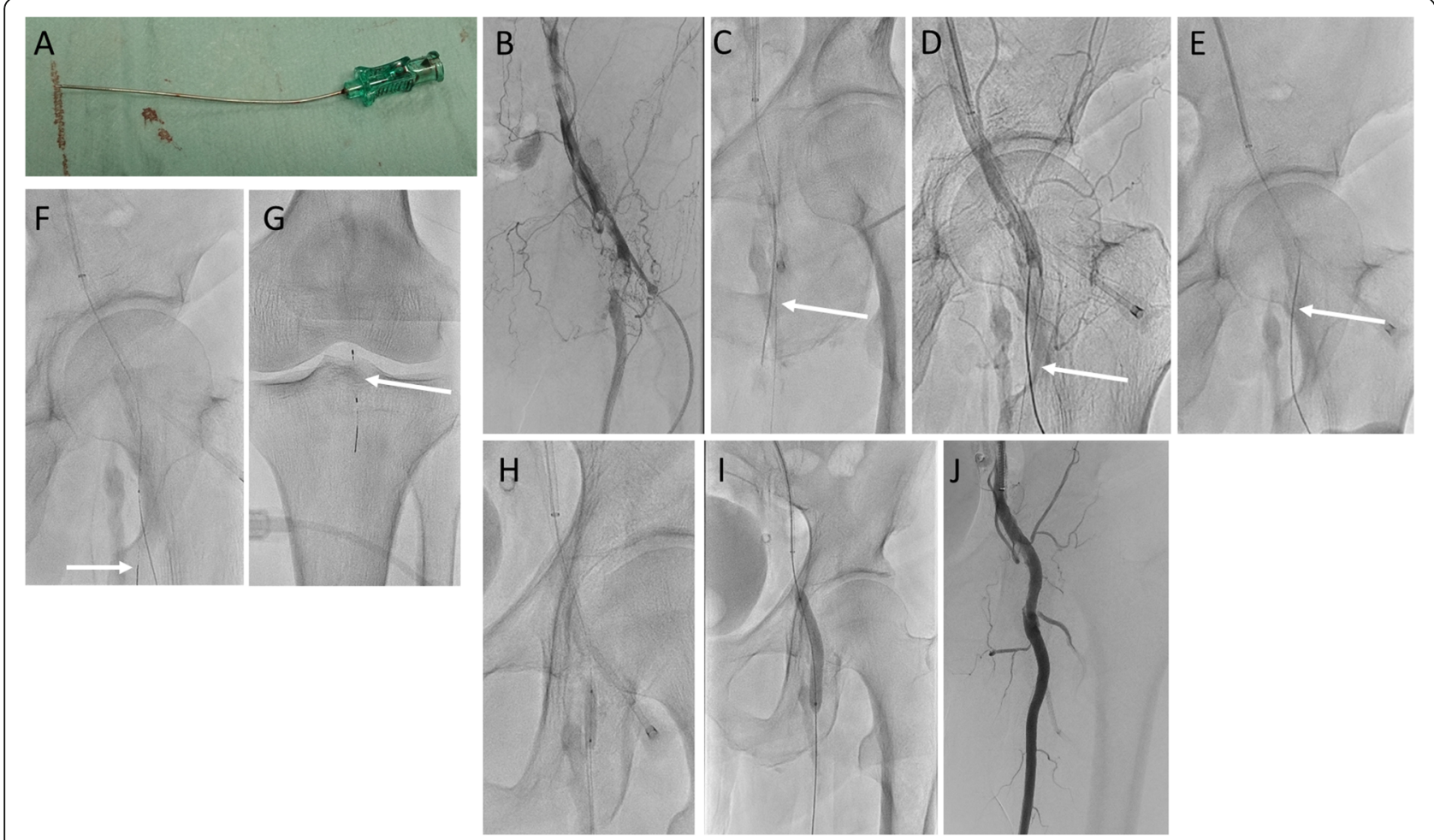

Fig. 4 a Image showing a 21-G needle that is slightly curved. b Calcified plaque in the left CFA. c, d The needle was advanced into the center of the calcified plaque while observing from multiple directions with a fluoroscopic guide. e The needle on the externalized guidewire is coaxially placed in and out several times to puncture the lesion. $\mathbf{f}$ An antegrade wire was advanced into the SFA. $\mathbf{g}$ We placed the filter wire in the popliteal artery. $\mathbf{h}$, $\mathbf{i}$ We dilated the CFA with a scoring balloon and high-pressure balloons. $\mathbf{j}$ Final angiography shows that the CFA is well opened

long-term patency is still unclear. A much larger study is required to confirm the safety and efficacy of our method. This procedure appears to be difficult to perform if the calcified plaques are continuous to the SFA and not just in the CFA, or if the SFA is completely occluded from just proximally. In such cases, a metal needle may be inserted directly from the distal part of the occluded CFA, only the CFA may pass through the calcified plaque by metal needle, and the remaining lesions may be passed through according to standard procedures. Even when the CFA to the external iliac artery (EIA) is continuously occluded, applying this technique appears to be difficult because the EIA has considerable bending and it is difficult to pass through it only with a needle. Additionally, applying this method to the ipsilateral up to the proximal SFA is possible. However, adapting to the direction of the mid-SFA or popliteal artery owing to the problem of needle length and angle is difficult. Although this technique may be applied to more peripheral arteries such as the dorsalis pedis artery, it seems that it is more difficult to pass the lesion by needle alone than CFA because of the small vessel diameter. To the best of our knowledge, this technique has not been previously reported. Therefore, more cases need to be accumulated and the efficacy and safety of this procedure need to be verified.

\section{Conclusions}

We have developed a useful technique for directly penetrating calcified plaques of the CFA using a bare metal needle for passage of a balloon and dilation of lesions. We call this approach the BAMBOO SPEAR technique. The BAMBOO SPEAR technique could be a useful option in EVT for an occlusive CFA with a calcified plaque.

\section{Abbreviations \\ CFA: Common femoral artery; EVT: Endovascular therapy; SFA: Superficial femoral artery; IVUS: Intravascular ultrasound; PAD: Peripheral artery disease; DCB: Drug coated balloon; CTO: Chronic total occlusion; EIA: External iliac artery}

\section{Acknowledgments}

We thank Ellen Knapp, PhD, from Edanz Group (https://en-author-services. edanzgroup.com/ac) for editing a draft of this manuscript.

\section{Authors' contributions}

$\mathrm{NH}, \mathrm{MA}, \mathrm{SH}, \mathrm{SS}$ : performed the procedure and performed pre and post procedure follow-up. SK: drafted the manuscript and revised it critically for important intellectual content. JK: gave final approval for the submitted manuscript. The authors read and approved the final manuscript. 


\section{Funding}

No specific grants from any funding agency in the public, commercial, or not-for-profit sectors were received for this study.

\section{Availability of data and materials}

The datasets used and/or analysed during the current study are available from the corresponding author on reasonable request.

\section{Declarations}

\section{Ethics approval and consent to participate}

All procedures were performed in accordance with the ethical standards of the institutional and/or national research committee and with the 1964 Declaration of Helsinki and its later amendments or comparable ethical standards.

\section{Consent for publication}

Written informed consent was obtained from the patient described in the case report.

\section{Competing interests}

The authors declare that they have no competing interests.

\section{Conflict of interest}

The authors declare that there is no conflict of interest regarding the publication of this article.

\section{Author details}

'Department of Cardiovascular Medicine, Asahi General Hospital, I-1326 Asahi, Chiba 289-2511, Japan. ${ }^{2}$ Department of Cardiovascular Medicine, University of Tokyo Hospital, Tokyo, Japan.

Received: 15 January 2021 Accepted: 24 February 2021

Published online: 04 March 2021

\section{References}

Gouëffic Y, Della Schiava N, Thaveau F et al (2017) Stenting or surgery for de novo common femoral artery stenosis. JACC Cardiovasc Interv 10:1344-1354

Ichihashi S, Sato T, Iwakoshi S et al (2014) Technique of percutaneous direct needle puncture of calcified plaque in the superficial femoral artery or tibial artery to facilitate balloon catheter passage and balloon dilation of calcified lesions. J Vasc Interv Radiol 25:784-788

Kuo TT, Chen PL, Huang CY et al (2019) Outcome of drug-eluting balloon angioplasty versus endarterectomy in common femoral artery occlusive disease. J Vasc Surg 69:141-147

Nakama T, Muraishi M, Obunai K et al (2020) Efficacy of the novel inner PIERCE technique for severely calcified below-the knee occlusions in a patient with chronic limb-threatening ischemia. Catheter Cardiovasc Interv. https://doi. org/10.1002/ccd.29255

Nguyen BN, Amdur RL, Abugideri M et al (2015) Postoperative complications after common femoral endarterectomy. J Vasc Surg 61:1489-1494

Nishibe T, Maruno K, Iwahori A et al (2015) The role of common femoral artery endarterectomy in the endovascular era. Ann Vasc Surg 29:1501-1507

Norgen L, Hiatt WR, Dormandy JA et al (2007) Inter-society consensus for the management of peripheral arterial disease (TASC II). J Vasc Surg 45:55-67. https://doi.org/10.1016/j.jejvs.2006.09.024

Stavroulakis J, Schwindt A, Torsello G et al (2018) Directional atherectomy with antirestenotic therapy vs drug-coated balloon angioplasty alone for common femoral artery atherosclerotic disease. J Endovasc Ther 25:92-99

\section{Publisher's Note}

Springer Nature remains neutral with regard to jurisdictional claims in published maps and institutional affiliations.

\section{Submit your manuscript to a SpringerOpen ${ }^{\circ}$ journal and benefit from:}

- Convenient online submission

- Rigorous peer review

- Open access: articles freely available online

High visibility within the field

- Retaining the copyright to your article

Submit your next manuscript at $\boldsymbol{\nabla}$ springeropen.com 